\title{
NORDHAUS - GADDUM TYPE RESULTS FOR WIENER LIKE INDICES OF GRAPHS
}

\author{
${ }^{1}$ R. BHUVANESHWARI, ${ }^{2}$. KALADEVI \\ ${ }^{1}$ Research Scholar (FT), PG and Research Department of Mathematics, Bishop Heber College, Trichy-620017, Tamil Nadu, India. \\ Email id: bhuvi950066@gmail.com. \\ ${ }^{2}$ Professor Emeritus, PG and Research Department of Mathematics, Bishop Heber College, Trichy-620017, Tamil Nadu, India. Email \\ id: kaladevi1956@gmail.com.
}

\section{ABSTRACT}

A Nordhaus - Gaddum type result is a lower or upper bound on sum or product of a parameter of a graph and its complement. This concept was introduced in 1956 by Nordhaus E. A., $\quad$ Gaddum J. W. Generalized Wiener like indices such as wiener index, Detour index, Reciprocal- wiener index, Harary- wiener index, Hyper- wiener index, Reciprocal- Detour index, Harary- Detour index and Hyper- Detour index have been studied in graph theory. In this paper, Nordhaus - Gaddum type results of these indices for k-Sun graph and four regular graph are presented.

Keywords: Generalized Wiener like Polynomial, k-sun graph, Nordhaus - Gaddum results.

\section{Introduction 1:}

All graphs considered in this paper are finite, simple and connected. For a graph $\mathrm{G}=(\mathrm{V}, \mathrm{E})$ with vertices $\mathrm{u}, \mathrm{v} \in \mathrm{V}$, the distance between $\mathrm{u}$ and $\mathrm{v}$ in $\mathrm{G}$, denoted by $d_{G}(u, v)$, is the length of a shortest $(u, v)$ - path in $\mathrm{G}$. The Wiener index $[2,3,4]$ of $\mathrm{G}$ is defined as $W(G)=\frac{1}{2} \sum_{u, v \in V(G)} d_{G}(u, v)$ with the summation going over all pairs of distinct vertices of $\mathrm{G}$. The above definition can be further generalized in the following way:

$W_{\lambda}(\mathrm{G})=\frac{1}{2} \sum_{u, v \in V(G)} d_{G}^{\lambda}(u, v)$ where

$d_{G}^{\lambda}(u, v)=\left(d_{G}(u, v)\right)^{\lambda}$

and $\lambda$ is any real number.

For particular instances of the invariant $\lambda, W_{-2}, W_{-1}$ and $\frac{1}{2} W_{1}+\frac{1}{2} W_{2}$ are the so called Harary index [1], reciprocal Wiener index and hyper - wiener index(WW) [5,6].

The detour index of $\mathrm{G}$ is defined as $D(G)=\frac{1}{2} \sum_{u, v \in V(G)} D_{G}(u, v)$ with the summation going over all pairs of distinct vertices of $\mathrm{G}$. The above definition can be further generalized in the following way:

$D_{\lambda}(\mathrm{G})=\frac{1}{2} \sum_{u, v \in V(G)} D_{G}^{\lambda}(u, v) \quad$ where $\quad D(u, v)=$ $\left(D_{G}(u, v)\right)^{\lambda}$ and $\lambda$ is any real number.

For particular instances of the invariant $\lambda, D_{-2}, D_{-1}$ and $\frac{1}{2} D_{1}+\frac{1}{2} D_{2}$ are the so called Harary detour index, reciprocal detour index and hyper - detour index(WW). The complement of a graph $\mathrm{G}$, denoted by $\bar{G}$ is the graph with the same vertex set as $G$, where two vertices in $G$ are adjacent if and only if they are not adjacent in $\mathrm{G}$.

\section{Definition 1.1:}

A $k-$ Sun graph $(k \geq 3)$ is the graph on $2 k$ vertices obtained from a clique $\mathrm{c}_{1}, \mathrm{c}_{2}, \ldots \mathrm{c}_{\mathrm{k}}$ on $\mathrm{k}$ vertices and an independent set on kertices. Let $\mathrm{V}(\mathrm{G})=\left\{\mathrm{c}_{1}, \mathrm{c}_{2}\right.$, $\left.\ldots \mathrm{c}_{\mathrm{k}}, \mathrm{s}_{1}, \mathrm{~s}_{2}, \ldots \mathrm{s}_{\mathrm{k}}\right\}$ and $\mathrm{E}(\mathrm{G})=\left\{\mathrm{sic}_{\mathrm{i}}, \mathrm{sic}_{\mathrm{i}+1} ; 1 \leq \mathrm{i} \leq \mathrm{k}\right\} \cup$ $\left\{\mathrm{s}_{\mathrm{k}} \mathrm{c}_{\mathrm{k}}, \mathrm{s}_{\mathrm{k}} \mathrm{c}_{1}\right\} \cup\left\{\mathrm{c}_{\mathrm{i}} \mathrm{c}_{\mathrm{j}} ; 1 \leq \mathrm{i} \leq \mathrm{k}, 1 \leq \mathrm{j} \leq \mathrm{k}, \mathrm{i}<\mathrm{j}\right\}$ be the vertex set and edge set of $\mathrm{G}$ respectively.

\subsection{Generalized Wiener like indices of $\mathbf{k}$ - sun graph and its complement graph:}




\section{Theorem 2.2.1:}

Let $\mathrm{G}$ be a $\mathrm{k}$ - sun graph. Then the generalized wiener polynomial, generalized detour polynomial and the generalized circular polynomial are given by

$W_{\lambda} P(G: x)=\left(\frac{k^{2}+3 k}{2}\right) x^{1^{\lambda}}+\left(k^{2}-k\right) x^{2^{\lambda}}+\left(\frac{k^{2}-3 k}{2}\right) x^{3^{\lambda}}$

$D_{\lambda} P(G: x)=\left(\frac{k^{2}-3 k}{2}\right) x^{(2 k-3)^{\lambda}}+\left(k^{2}-k\right) x^{(2 k-2)^{\lambda}}+\left(\frac{k^{2}+3 k}{2}\right) x^{(2 k-1)^{\lambda}}$, and

$C_{\lambda} P(G: x)=\left(\frac{k^{2}-3 k}{2}\right) x^{(2 k-2)^{\lambda}}+k x^{(2 k-1)^{\lambda}}+k^{2} x^{2 k^{\lambda}}+k x^{(2 k+1)^{\lambda}}+\left(\frac{k^{2}-3 k}{2}\right) x^{(2 k+2)^{\lambda}}$,

where $\mathrm{k} \geq 4$ and $\lambda$ is any real number.

\section{Proof:}

Let $\mathrm{G}$ be $\mathrm{k}-$ sun graph on $2 \mathrm{k}$ vertices, where $\mathrm{k} \geq 4$ and $\lambda$ is any real number.

Let $\mathrm{V}(\mathrm{G})=\left\{\mathrm{c}_{1}, \mathrm{c}_{2}, \ldots \mathrm{c}_{\mathrm{k}}, \mathrm{s}_{1}, \mathrm{~s}_{2}, \ldots \mathrm{s}_{\mathrm{k}}\right\}$ and $\mathrm{E}(\mathrm{G})=\left\{\mathrm{s}_{\mathrm{i}} \mathrm{c}_{\mathrm{i}}\right.$, $\left.\mathrm{sic}_{\mathrm{i}} \mathrm{c}_{\mathrm{i}+1} ; 1 \leq \mathrm{i} \leq \mathrm{k}\right\} \cup\left\{\mathrm{s}_{\mathrm{k}} \mathrm{c}_{\mathrm{k}}, \mathrm{s}_{\mathrm{k}} \mathrm{c}_{1}\right\} \cup\left\{\mathrm{c}_{\mathrm{i}} \mathrm{c}_{\mathrm{j}} ; 1 \leq \mathrm{i} \leq \mathrm{k}, 1 \leq \mathrm{j}\right.$ $\leq \mathrm{k}, \mathrm{i}<\mathrm{j}\}$ be the vertex set and edge set of $\mathrm{G}$ respectively. The generalized Wiener like polynomial of $\mathrm{G}$ is defined as, $W_{\lambda} P(G: x)=\sum_{u, v \in V(G)} x^{d^{\lambda}(u \cdot v)}$, for any real number $\lambda$.

For the k - sun graph, the generalized wiener polynomial, the generalized detour polynomial and generalized circular polynomial of $\mathrm{k}$ - sun graph $\mathrm{G}$ are,

$$
\begin{aligned}
& W_{\lambda} P(G: x)=\sum_{1 \leq i<j \leq k} x^{d^{\lambda}\left(c_{i} c_{j}\right)}+\sum_{1 \leq i \leq k} x^{d^{\lambda}\left(c_{i} s_{i}\right)}+\sum_{1 \leq i<j \leq k} x^{d^{\lambda}\left(s_{i} s_{j}\right)}---(1) \\
& D_{\lambda} P(G: x)=\sum_{1 \leq i<j \leq k} x^{D^{\lambda}\left(c_{i} c_{j}\right)}+\sum_{1 \leq i \leq k} x^{D^{\lambda}\left(c_{i} s_{i}\right)}+\sum_{1 \leq i<j \leq k} x^{D^{\lambda}\left(s_{i} s_{j}\right)}----(2) \\
& C_{\lambda} P(G: x)=\sum_{1 \leq i<j \leq k} x^{C^{\lambda}\left(c_{i} c_{j}\right)}+\sum_{1 \leq i \leq k} x^{C^{\lambda}\left(c_{i} s_{i}\right)}+\sum_{1 \leq i<j \leq k} x^{C^{\lambda}\left(s_{i} s_{j}\right)}---(3)
\end{aligned}
$$

The 4-sun graph and the wiener detour matrix are shown in Figure 1.1 and Figure 1.2 respectively. Figure 1.3 shows the circular matrix of the $4-$ Sun graph. The wiener-detour matrix and the circular matrix gives the wiener polynomial, the detour polynomial and circular polynomial of the $4-$ s-un graph. 


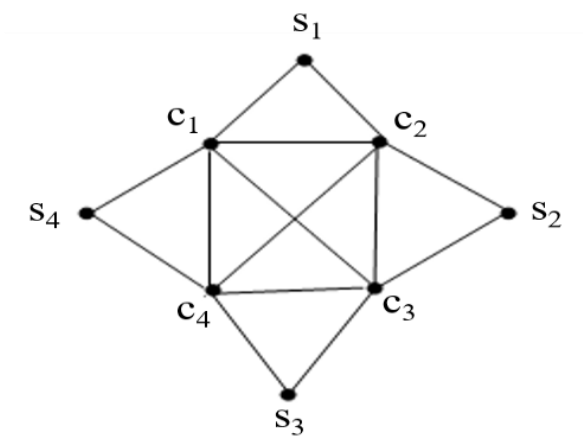

Figure 1.1 4-Sun Graph.

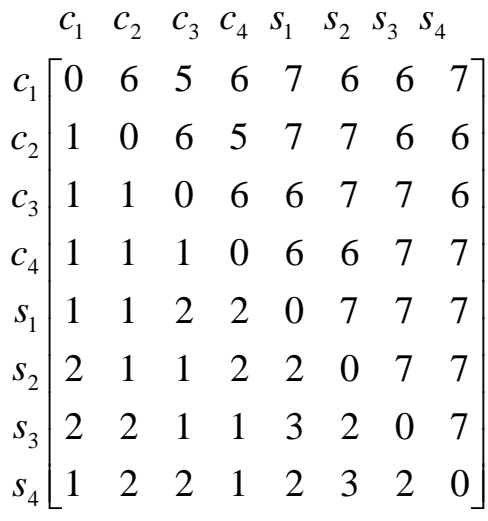

Figure 1.2 WDM(4-Sun graph)

$$
\begin{aligned}
& \begin{array}{llllllll}
\mathrm{c}_{1} & \mathrm{c}_{2} & \mathrm{c}_{3} & \mathrm{c}_{4} & \mathrm{~s}_{1} & \mathrm{~s}_{2} & \mathrm{~s}_{3} & \mathrm{~s}_{4}
\end{array}
\end{aligned}
$$

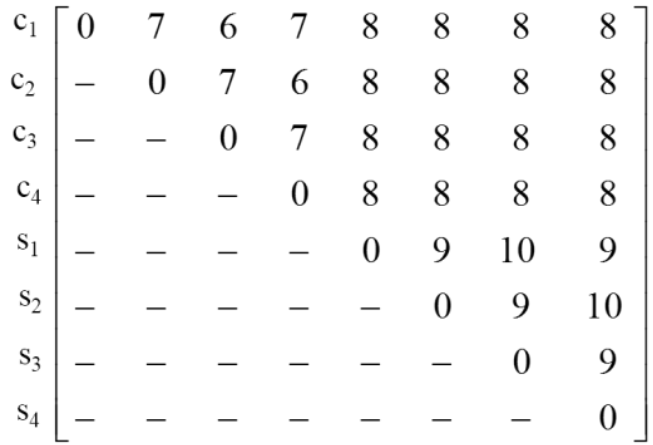

Figure 1.3 CM(4-Sun graph)

$W_{\lambda} P(G: x)=14 \mathrm{x}^{1^{12}}+12 \mathrm{x}^{2^{2}}+2 \mathrm{x}^{3^{2}} ; D_{\lambda} P(G: x)=2 \mathrm{x}^{\mathrm{5}^{2}}+12 \mathrm{x}^{6^{2}}+14 \mathrm{x}^{7^{2}} ; C_{\lambda} P(G: x)=2 \mathrm{x}^{6^{2}}+4 \mathrm{x}^{7^{2}}+16 \mathrm{x}^{8^{2}}+4 \mathrm{x}^{9^{2}}+2 \mathrm{x}^{10^{2}}$.

The 5-sun graph and the wiener detour matrix are shown in Figure 1.4 and Figure 1.5 respectively. Figure 1.6 shows the circular matrix of the 5 - sun graph. The wiener-detour matrix and the circular

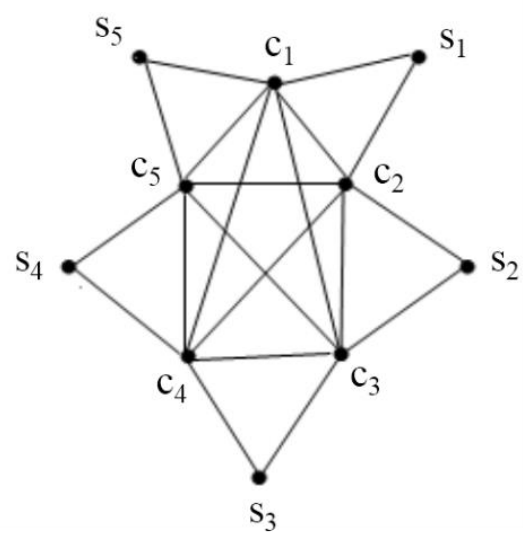

Figure 1.4 5-Sun Graph. matrix gives the wiener polynomial, the detour polynomial and circular polynomial of the 5 - sun graph.

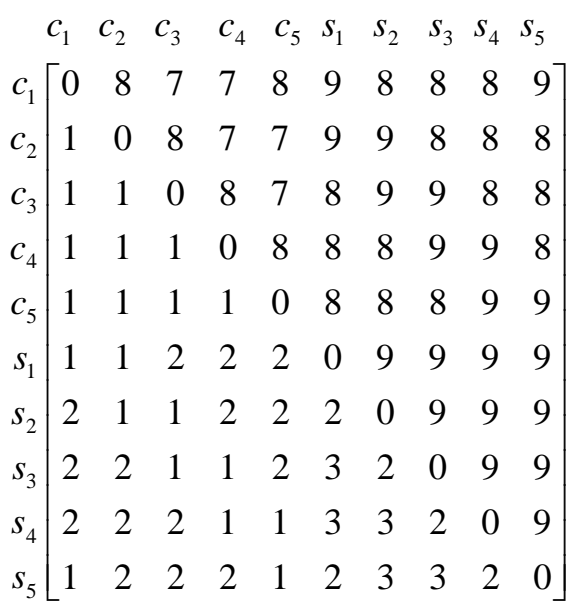

Figure 1.5 WDM(5-Sun graph) 


\begin{tabular}{|c|c|c|c|c|c|c|c|c|c|c|}
\hline 1 & $\begin{array}{l}c_{1} \\
0\end{array}$ & $\begin{array}{l}c_{2} \\
9\end{array}$ & $\begin{array}{c}c_{3} \\
8\end{array}$ & $\begin{array}{c}c_{4} \\
8\end{array}$ & $\begin{array}{c}c_{5} \\
9\end{array}$ & 10 & 10 & $\begin{array}{c}s_{3} \\
10\end{array}$ & $\begin{array}{l}\mathrm{S}_{4} \\
10\end{array}$ & $\begin{array}{l}3_{5} \\
10\end{array}$ \\
\hline & - & 0 & 9 & 8 & 8 & 10 & 10 & 10 & 10 & 10 \\
\hline & - & - & 0 & 9 & 8 & 10 & 10 & 10 & 10 & 10 \\
\hline & - & - & - & 0 & 9 & 10 & 10 & 10 & 10 & 10 \\
\hline & - & - & - & - & 0 & 10 & 10 & 10 & 10 & 10 \\
\hline & - & - & - & - & - & 0 & 11 & 12 & 12 & 11 \\
\hline & - & - & - & - & - & - & 0 & 11 & 12 & 12 \\
\hline & - & - & - & - & - & - & - & 0 & 11 & 12 \\
\hline & - & - & - & - & - & - & - & - & 0 & 11 \\
\hline & & & - & - & - & - & - & - & - & 0 \\
\hline
\end{tabular}

Figure 1.6 CM(5-Sun graph)

$W_{\lambda} P(G: x)=20 \mathrm{x}^{1^{\lambda}}+20 \mathrm{x}^{2^{\lambda}}+5 \mathrm{x}^{3^{\lambda}} ; D_{\lambda} P(G: x)=5 \mathrm{x}^{7^{\lambda}}+20 \mathrm{x}^{8^{\lambda}}+20 \mathrm{x}^{9^{\lambda}} ; C_{\lambda} P(G: x)=5 \mathrm{x}^{8^{\lambda}}+5 \mathrm{x}^{9^{\lambda}}+25 \mathrm{x}^{10^{\lambda}}+5 \mathrm{x}^{11^{\lambda}}+5 \mathrm{x}^{12^{\lambda}}$. For $\mathrm{k}=6$, the corresponding wiener polynomial, detour polynomial and polynomial of 6 - sun graph given below, $W_{\lambda} P(G: x)=27 \mathrm{x}^{1^{\lambda}}+30 \mathrm{x}^{2^{\lambda}}+9 \mathrm{x}^{3^{\lambda}} ; D_{\lambda} P(G: x)=9 \mathrm{x}^{9^{\lambda}}+30 \mathrm{x}^{10^{\lambda}}+27 \mathrm{x}^{11^{\lambda}} ; C_{\lambda} P(G: x)=9 \mathrm{x}^{10^{\lambda}}+6 \mathrm{x}^{11^{\lambda}}+36 \mathrm{x}^{12^{\lambda}}+6 \mathrm{x}^{13^{\lambda}}+9 \mathrm{x}^{14^{\lambda}}$.

For $\mathrm{k}=7$, the corresponding wiener polynomial, detour polynomial and polynomial of 7 - sun graph given below, $W_{\lambda} P(G: x)=35 \mathrm{x}^{1^{\lambda}}+42 \mathrm{x}^{2^{\lambda}}+14 \mathrm{x}^{3^{\lambda}} ; D_{\lambda} P(G: x)=14 \mathrm{x}^{11^{\lambda}}+42 \mathrm{x}^{12^{\lambda}}+35 \mathrm{x}^{13^{\lambda}} ; C_{\lambda} P(G: x)=14 \mathrm{x}^{12^{\lambda}}+7 \mathrm{x}^{13^{\lambda}}+49 \mathrm{x}^{14^{\lambda}}+7 \mathrm{x}^{15^{\lambda}}+14 \mathrm{x}^{16^{\lambda}}$.

For $\mathrm{k}=8$, the corresponding wiener polynomial, detour polynomial and polynomial of 8 - sun graph given below, $W_{\lambda} P(G: x)=44 \mathrm{x}^{1^{\lambda}}+56 \mathrm{x}^{2^{\lambda}}+20 \mathrm{x}^{3^{\lambda}} ; D_{\lambda} P(G: x)=20 \mathrm{x}^{13^{\lambda}}+56 \mathrm{x}^{14^{\lambda}}+44 \mathrm{x}^{15^{\lambda}} ; C_{\lambda} P(G: x)=20 \mathrm{x}^{14^{\lambda}}+8 \mathrm{x}^{15^{\lambda}}+64 \mathrm{x}^{16^{\lambda}}+8 \mathrm{x}^{17^{\lambda}}+20 \mathrm{x}^{18^{\lambda}}$.

Hence in general, the generalized wiener polynomial, the detour polynomial and the generalized circular polynomial of $\mathrm{k}$ - sun graph $\mathrm{G}$ are given by,

$$
\begin{aligned}
& W_{\lambda} P(G: x)=\left(\frac{k^{2}+3 k}{2}\right) x^{1^{\lambda}}+\left(k^{2}-k\right) x^{2^{\lambda}}+\left(\frac{k^{2}-3 k}{2}\right) x^{3^{\lambda}} \\
& D_{\lambda} P(G: x)=\left(\frac{k^{2}-3 k}{2}\right) x^{(2 k-3)^{\lambda}}+\left(k^{2}-k\right) x^{(2 k-2)^{\lambda}}+\left(\frac{k^{2}+3 k}{2}\right) x^{(2 k-1)^{\lambda}} \\
& C_{\lambda} P(G: x)=\left(\frac{k^{2}-3 k}{2}\right) x^{(2 k-2)^{\lambda}}+k x^{(2 k-1)^{\lambda}}+k^{2} x^{2 k^{\lambda}}+k x^{(2 k+1)^{\lambda}}+\left(\frac{k^{2}-3 k}{2}\right) x^{(2 k+2)^{\lambda}}
\end{aligned}
$$

\section{Corollary 2.2.2:}

Let $\mathrm{G}$ be a $\mathrm{k}$-sun graph for $\mathrm{k} \geq 4$. Then, the Wiener index $\mathrm{W}_{1}(\mathrm{G})=4 \mathrm{k}^{2}-5 \mathrm{k}$,

The Reciprocal-Wiener index $\mathrm{W}_{-1}(\mathrm{G})=-\left[4 \mathrm{k}^{2}-5 \mathrm{k}\right]$, The Harary-Wiener index $\mathrm{W}_{-2}(\mathrm{G})=-2\left[4 \mathrm{k}^{2}-5 \mathrm{k}\right]$,
The Hyper-Wiener index WW(G) $=\frac{1}{2}\left[12 \mathrm{k}^{2}-15 \mathrm{k}\right]$.

Corollary 2.2.3:

Let $\mathrm{G}$ be a $\mathrm{k}$ - sun graph for $\mathrm{k} \geq 4$. Then 
The Detour index $D_{1}(G)=\left[4 k^{3}-6 k^{2}+5 k\right]$, The Reciprocal Detour index $D_{-1}(G)=-\left[4 k^{3}-6 k^{2}+5 k\right]$,

The Harary - Detour index $D_{-2}(G)=-2\left[4 k^{3}-6 k^{2}+5 k\right]$, The Hyper - Detour index $D D(G)=\frac{1}{2}\left[12 k^{3}-18 k^{2}+15 k\right]$.

\section{Theorem 2.2.4:}

Let $\mathrm{G}$ be the $\mathrm{k}$ - sun graph on $2 \mathrm{k}$ vertices. Let $\bar{G}$ be Let $\bar{G}$ be the complement of $\mathrm{k}$ - sun graph $\mathrm{G}$. Then the generalized wiener polynomial and detour polynomial for $\bar{G}$ are respectively given by: the complement of k - sun graph G. Figure 1.7. shows the complement graph $\bar{G}$ of 5 - sun graph. The wiener detour matrix of the complement of 5 - Sun graph in Figure 1.8. gives the wiener polynomial and

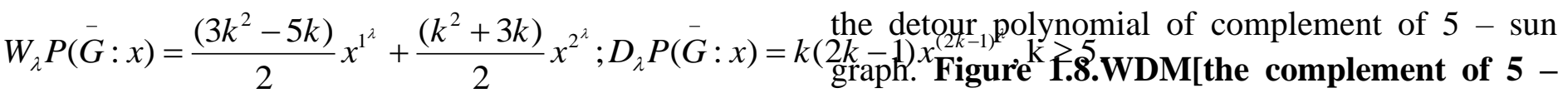
Proof:

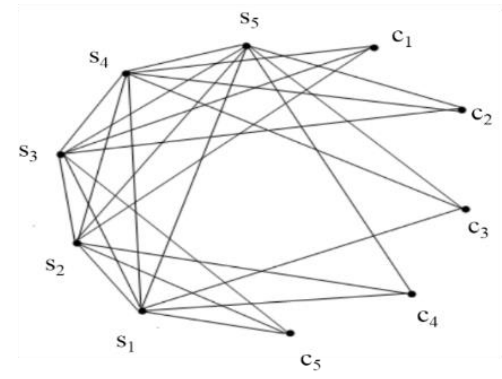

Figure $1.7 \bar{G}(5-$ sun graph $)$

\begin{tabular}{|c|c|c|c|c|c|c|c|c|c|c|}
\hline \multicolumn{2}{|c|}{$\mathrm{c}_{1}$} & $c_{2}$ & $c_{3}$ & $\mathrm{c}_{4}$ & $\mathrm{c}_{5}$ & $\mathrm{~s}_{1}$ & $\mathrm{~s}_{2}$ & $\mathrm{~S}_{3}$ & & \\
\hline $\mathrm{c}_{1}$ & 0 & 9 & 9 & 9 & 9 & 9 & 9 & 9 & & 9 \\
\hline & 2 & 0 & 9 & 9 & 9 & 9 & 9 & 9 & 9 & 0 \\
\hline 3 & 2 & 2 & 0 & 9 & 9 & 9 & 9 & 9 & 9 & 9 \\
\hline 4 & 2 & 2 & 2 & 0 & 9 & 9 & 9 & 9 & 9 & 9 \\
\hline 5 & 2 & 2 & 2 & 2 & 0 & 9 & 9 & 9 & 0 & O \\
\hline$s_{1}$ & 2 & 1 & 1 & 1 & 2 & 0 & 9 & 9 & 9 & 9 \\
\hline$s_{2}$ & 1 & 2 & 1 & 1 & 1 & 1 & 0 & 9 & 9 & 9 \\
\hline$s_{3}$ & 1 & 2 & 2 & 1 & 1 & 1 & 1 & 0 & 9 & 9 \\
\hline$s_{4}$ & 1 & 1 & 2 & 2 & 1 & 1 & 1 & 1 & 0 & 9 \\
\hline $\mathrm{s}_{5}$ & 2 & 1 & 1 & 2 & 2 & 1 & 1 & 1 & & \\
\hline
\end{tabular}

Figure 1.8 WDM $(\bar{G}(5-\operatorname{sun}$ graph $))$

$W_{\lambda} P(\stackrel{-}{G}: x)=25 x^{1^{\lambda}}+20 x^{2^{\lambda}} ; D_{\lambda} P(\stackrel{-}{G}: x)=45 x^{9^{\lambda}}$

The wiener detour matrix of the complement of 6 - Sun graph in Figure 1.9 gives the wiener polynomial and the detour polynomial of complement of 6 - sun graph. 


\begin{tabular}{|c|c|c|c|c|c|c|c|c|c|c|c|c|}
\hline & & $c_{2}$ & $c_{3}$ & & & & $\mathrm{~s}_{1}$ & $s_{2}$ & $\mathrm{~s}_{3}$ & $\mathrm{~s}_{4}$ & $\mathrm{~s}_{5}$ & $\mathrm{~s}_{6}$ \\
\hline & 0 & 11 & 11 & 11 & 11 & 11 & 11 & 11 & 11 & 11 & 11 & 117 \\
\hline & 2 & 0 & 11 & 11 & 11 & 11 & 11 & 11 & 11 & 11 & 11 & 11 \\
\hline & 2 & 2 & 0 & 11 & 11 & 11 & 11 & 11 & 11 & 11 & 11 & 11 \\
\hline & 2 & 2 & 2 & 0 & 11 & 11 & 11 & 11 & 11 & 11 & 11 & 11 \\
\hline & 2 & 2 & 2 & 2 & 0 & 11 & 11 & 11 & 11 & 11 & 11 & 11 \\
\hline$\because 6$ & 2 & 2 & 2 & 2 & 2 & 0 & 11 & 11 & 11 & 11 & 11 & 11 \\
\hline $\mathrm{s}_{1}$ & 2 & 1 & 1 & 1 & 1 & 2 & 0 & 11 & 11 & 11 & 11 & 11 \\
\hline$\xi_{2}$ & 1 & 2 & 1 & 1 & 1 & 1 & 1 & 0 & 11 & 11 & 11 & 11 \\
\hline$s_{3}$ & 1 & 2 & 2 & 1 & 1 & 1 & 1 & 1 & 0 & 11 & 11 & 11 \\
\hline$s_{4}$ & 1 & 1 & 2 & 2 & 1 & 1 & 1 & 1 & 1 & 0 & 11 & 11 \\
\hline$s_{5}$ & 1 & 1 & 1 & 2 & 2 & 1 & 1 & 1 & 1 & 1 & 0 & 11 \\
\hline & 2 & 1 & 1 & 1 & 2 & 2 & 1 & 1 & 1 & 1 & 1 & 0 \\
\hline
\end{tabular}

\section{Figure 1.9 WDM[the complement of 6 - Sun graph]}

$W_{\lambda} P(\bar{G}: x)=39 x^{1^{\lambda}}+27 x^{2^{\lambda}} ; D_{\lambda} P(\bar{G}: x)=66 x^{11^{\lambda}}$

For $\mathrm{k}=7,8,9$ the corresponding wiener polynomials and detour polynomial of the complement of $\mathrm{k}-$ sun graph given below,

$W_{\lambda} P(\bar{G}: x)=56 x^{1^{\lambda}}+35 x^{2^{\lambda}} ; W_{\lambda} P(\bar{G}: x)=76 x^{1^{\lambda}}+44 x^{2^{\lambda}} ; W_{\lambda} P(\bar{G}: x)=90 x^{1^{\lambda}}+54 x^{2^{\lambda}}$

$D_{\lambda} P(\vec{G}: x)=91 x^{13^{\lambda}} ; D_{\lambda} P(\vec{G}: x)=120 x^{15^{\lambda}} ; D_{\lambda} P(\vec{G}: x)=153 x^{17^{\lambda}}$

Hence in general, the generalized wiener and detour polynomial of complement of $\mathrm{k}$ - sun graph are respectively given by,

$W_{\lambda} P(\bar{G}: x)=\frac{\left(3 k^{2}-5 k\right)}{2} x^{1^{\lambda}}+\frac{\left(k^{2}+3 k\right)}{2} x^{2^{\lambda}} ; D_{\lambda} P(\bar{G}: x)=k(2 k-1) x^{(2 k-1)^{\lambda}}, \mathrm{k} \geq 5 .$.

\section{Corollary 2.2.5:}

Let $\bar{G}$ be the complement of $\mathrm{k}$ - sun graph $\mathrm{G}$, then

The Wienerindex $W_{1}(\vec{G})=\frac{1}{2}\left[5 k^{2}+k\right] ;$ The Reciprocalindex $W_{-1}(\stackrel{-}{G})=-\frac{1}{2}\left[5 k^{2}+k\right]$;

The Harary - Wiener index $W_{-2}(\stackrel{-}{G})=-\frac{1}{2}\left[10 k^{2}+2 k\right]$; The Hyper - Wiener index $W W(\bar{G})=\frac{1}{4}\left[15 k^{2}+3 k\right]$

\section{Corollary 2.2.6:}

Let $\bar{G}$ be the complement of $\mathrm{k}$ - sun graph $\mathrm{G}$, then

The Detour index $D_{1}(\bar{G})=4 k^{3}-4 k^{2}+k$; The Reciprocal - Detour index $D_{-1}(\bar{G})=-\left[4 k^{3}-4 k^{2}+k\right]$

The Harary - Detour index $D_{-2}(\bar{G})=-2\left[4 k^{3}-4 k^{2}+k\right]$; The Hyper - Detour index $D D(\bar{G})=\frac{\left[12 k^{3}-12 k^{2}+3 k\right]}{2}$

Result 2.2.7: Nordhaus - Gaddum Equations of $\mathrm{k}$ - sun graph. 
$(i) W_{1}(G)+W_{1}(\bar{G})=\frac{13 k^{2}-9 k}{2} ;(i i) W_{-1}(G)+W_{-1}(\bar{G})=-\left[\frac{13 k^{2}-9 k}{2}\right]$

$($ iii $) W_{-2}(G)+W_{-2}(\stackrel{-}{G})=-\left[13 k^{2}-9 k\right] ;(i v) W W(G)+W W(-\bar{G})=\frac{39 k^{2}-27 k}{4}$

$(v) D_{1}(G)+D_{1}(\vec{G})=\left[8 k^{3}-10 k^{2}+6 k\right] ;(v i) D_{-1}(G)+D_{-1}(\vec{G})=-\left[8 k^{3}-10 k^{2}+6 k\right]$

$($ vii $) D_{-2}(G)+D_{-2}(\bar{G})=-2\left[8 k^{3}-10 k^{2}+6 k\right] ;(v i i i) D D(G)+D D(\bar{G})=\frac{24 k^{3}-30 k^{2}+18 k}{2}$

\subsection{Nordhaus - Gaddum Equation for four regular graph:}

In this section the generalized wiener polynomial and generalized detour polynomial of four regular graph and complement of four regular graph are presented and also Nordhaus - Gaddum equation for four regular graph is derived.

\section{Algorithm for Four regular graph:}

Input : the number of vertices $n$ of a cyclic graph.

Output : the class four regular graph with $2 \mathrm{n}$ vertices.

Begin

Step 1: Take a cycle $C_{n}$ with vertex set $V=\left\{v_{1}, v_{2}, \ldots v_{n}\right\}$ and e dge set $E=\left\{v_{i} v_{i+1} U v_{n} v_{1}\right.$ :

$1 \leq \mathrm{i} \leq(\mathrm{n}-1)\}$.

Step 2: For the edge $v_{i} V_{i+1}, 1 \leq i \leq(n-1)$ introduce a new vertex $u_{i}$ and create new edge $v_{i} u_{i}$ and $\mathrm{V}_{\mathrm{i}-1} \mathrm{u}_{\mathrm{i}}$.

Step 3: For the edge $\mathrm{v}_{\mathrm{n}} \mathrm{v}_{1}$ introduce a new vertex $\mathrm{u}_{\mathrm{n}}$ and create new edges $\mathrm{v}_{\mathrm{n}} \mathrm{u}_{\mathrm{n}}$ and $\mathrm{v}_{1} \mathrm{u}_{\mathrm{n}}$.

Step 4: From the set of new vertices $u_{i}$, create new edges $u_{i} u_{i+1}$ for $1 \leq i \leq(n-1)$ and an edge $u_{n} u_{1}$.

Step 5: The new four regular graph $\mathrm{G}\left(\mathrm{C}_{\mathrm{n}}\right)=(\mathrm{V}, \mathrm{E})$ has the vertex set and edge set

$\mathrm{V}_{\mathrm{G}}=\left\{\mathrm{v}_{1}, \mathrm{v}_{2}, \ldots \mathrm{v}_{\mathrm{n}}, \mathrm{u}_{1}, \mathrm{u}_{2}, \ldots \mathrm{u}_{\mathrm{n}}\right\}$

$E_{G}=\left\{u_{i} v_{i+1}, v_{n} v_{1}, v_{i} u_{i}, v_{i+1} u_{i}, v_{n} u_{n}, v_{1} u_{n}, u_{i} u_{i+1}, u_{n} u_{1} / 1 \leq i \leq(n-1)\right\}$.

\section{Generalized Wiener like indices of four regular graph and its complement:}

\section{Theorem 3.1.1:}

Let $G\left(C_{n}\right)$ be a four regular graph. Then the generalized wiener polynomial and the generalized detour polynomial are given by the following expressions:

$W_{\lambda} P(G: x)=4 n x^{1^{\lambda}}+4 n x^{2^{\lambda}}+\ldots . .+4 n x^{\left(\frac{n-1}{2}\right)^{\lambda}}+n x^{\left(\frac{n+1}{2}\right)^{\lambda}}$, when $\mathrm{n}$ is odd and $\mathrm{n} \geq 3$.

$W_{\lambda} P(G: x)=4 n x^{1^{\lambda}}+4 n x^{2^{\lambda}}+\ldots . .+4 n x^{\left(\frac{n-2}{2}\right)^{\lambda}}+3 n x^{\left(\frac{n}{2}\right)^{\lambda}}$, when $\mathrm{n}$ is even and $\mathrm{n} \geq 4$.

$D_{\lambda} P(G: x)=\frac{n(n-1)}{2} x^{(n-1)^{\lambda}}$

\section{Proof:}

Let $\mathrm{G}=\mathrm{G}\left(\mathrm{C}_{\mathrm{n}}\right)$ be a four regular graph with $2 \mathrm{n}$ vertices. Let $\mathrm{V}(\mathrm{G})=\left\{\mathrm{v}_{1}, \mathrm{v}_{2}, \ldots \mathrm{v}_{\mathrm{n}}\right\}$ and edge set

$\mathrm{E}=\left\{\mathrm{u}_{\mathrm{i}} \mathrm{v}_{\mathrm{i}+1}, \mathrm{v}_{\mathrm{n}} \mathrm{v}_{1}, \mathrm{v}_{\mathrm{i}} \mathrm{u}_{\mathrm{i}}, \mathrm{v}_{\mathrm{i}+1} \mathrm{u}_{\mathrm{i}}, \mathrm{v}_{\mathrm{n}} \mathrm{u}_{\mathrm{n}}, \mathrm{v}_{1} \mathrm{u}_{\mathrm{n}}, \mathrm{u}_{\mathrm{i}} \mathrm{u}_{\mathrm{i}+1}, \mathrm{u}_{\mathrm{n}} \mathrm{u}_{1} / 1 \leq \mathrm{i} \leq(\mathrm{n}-1)\right\}$.

Case(i): When $n$ is odd. 
Figure 1.10. shows the four regular graph $\mathrm{G}\left(\mathrm{C}_{3}\right)$ and Figure 1.11. gives the wiener polynomial and the detour polynomial of four regular graph $\mathrm{G}\left(\mathrm{C}_{3}\right)$.

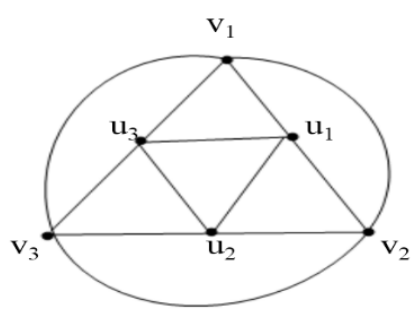

Figure $1.10 \mathrm{G}\left(\mathbf{C}_{3}\right)$

\begin{tabular}{|c|c|c|c|c|}
\hline v1 & $\mathrm{v}_{2}$ & V3 & $\mathrm{u}_{1}$ & $\mathrm{u}_{2}$ \\
\hline 0 & 5 & 5 & 5 & 5 \\
\hline 1 & 0 & 5 & 5 & 5 \\
\hline 1 & 1 & 0 & 5 & 5 \\
\hline 1 & 1 & 2 & 0 & 5 \\
\hline 2 & 1 & 1 & 1 & 0 \\
\hline 1 & 2 & 1 & 1 & 1 \\
\hline
\end{tabular}

Figure 1.11.WDM[G(C3)]

$W_{\lambda} P(G: x)=12 x^{1^{\lambda}}+3 x^{2^{\lambda}} ; D_{\lambda} P(G: x)=15 x^{5^{\lambda}}$

Figure 1.12. shows the four regular graph $\mathrm{G}\left(\mathrm{C}_{5}\right)$ and Figure 1.13. gives the wiener polynomial and the detour polynomial of four regular graph $\mathrm{G}\left(\mathrm{C}_{5}\right)$.

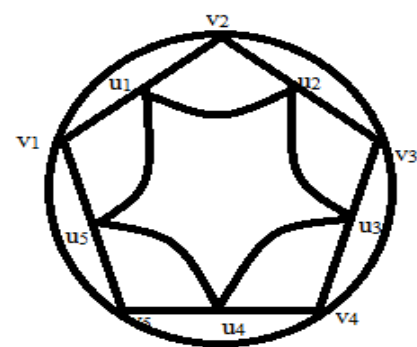

Figure 1.12 G(C5)

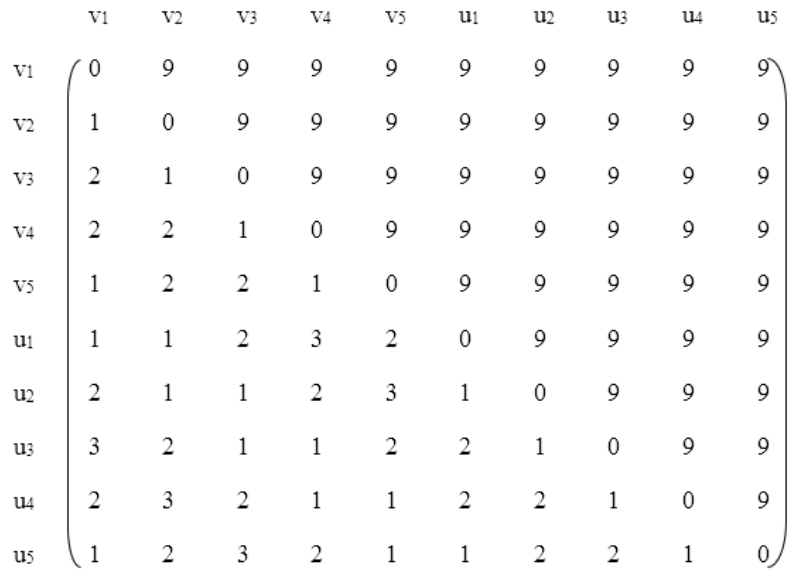

Figure 1.13.WDM[G(C5)]

$W_{\lambda} P(G: x)=20 x^{1^{\lambda}}+20 x^{2^{\lambda}}+5 x^{3^{\lambda}} ; D_{\lambda} P(G: x)=45 x^{9^{\lambda}}$

Hence in general, the generalized wiener polynomial of four regular graph $G\left(C_{n}\right)$ is,

$W_{\lambda} P(G: x)=4 n x^{1^{\lambda}}+4 n x^{2^{\lambda}}+\ldots . .+4 n x^{\left(\frac{n-1}{2}\right)^{\lambda}}+n x^{\left(\frac{n+1}{2}\right)^{\lambda}}$, when $\mathrm{n}$ is odd and $\mathrm{n} \geq 3$.

Case (ii): When $\mathrm{n}$ is even.

Figure 1.14. shows the four regular graph $\mathrm{G}\left(\mathrm{C}_{4}\right)$ and Figure 1.15. gives the wiener polynomial and the detour polynomial of four regular graph $\mathrm{G}\left(\mathrm{C}_{4}\right)$. 


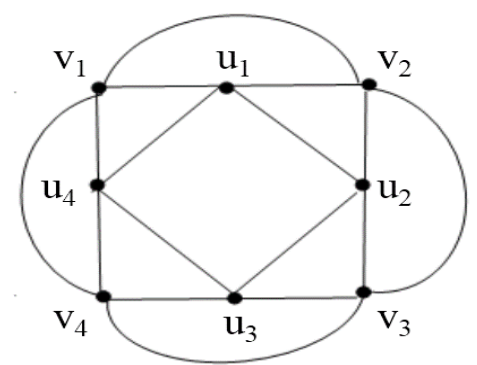

Figure 1.14. $\mathbf{G}\left(\mathrm{C}_{4}\right)$

\begin{tabular}{|c|c|c|c|c|c|c|c|}
\hline $\mathrm{v} 1$ & $\mathrm{v}_{2}$ & v3 & $\mathrm{v}_{4}$ & $\mathrm{u}_{1}$ & $\mathrm{u}_{2}$ & $\mathrm{u}_{3}$ & $u_{4}$ \\
\hline$(0$ & 7 & 7 & 7 & 7 & 7 & 7 & $7)$ \\
\hline 1 & 0 & 7 & 7 & 7 & 7 & 7 & 7 \\
\hline 2 & 1 & 0 & 7 & 7 & 7 & 7 & 7 \\
\hline 1 & 2 & 1 & 0 & 7 & 7 & 7 & 7 \\
\hline 1 & 1 & 2 & 2 & 0 & 7 & 7 & 7 \\
\hline 2 & 1 & 1 & 2 & 1 & 0 & 7 & 7 \\
\hline 2 & 2 & 1 & 1 & 2 & 1 & 0 & 7 \\
\hline 1 & 2 & 2 & 1 & 1 & 2 & 1 & 0 \\
\hline
\end{tabular}

Figure 1.15.WM[G(C4)]

$W_{\lambda} P(G: x)=16 x^{1^{\lambda}}+12 x^{2^{\lambda}} ; D_{\lambda} P(G: x)=28 x^{7^{\lambda}}$.

Hence in general, the generalized wiener polynomial of four regular graph $\mathrm{G}\left(\mathrm{C}_{\mathrm{n}}\right)$ is $W_{\lambda} P(G: x)=4 n x^{1^{\lambda}}+4 n x^{2^{\lambda}}+\ldots . .+4 n x^{\left(\frac{n-1}{2}\right)^{\lambda}}+n x^{\left(\frac{n+1}{2}\right)^{\lambda}}$, when $\mathrm{n}$ is odd and $\mathrm{n} \geq 3$.

$W_{\lambda} P(G: x)=4 n x^{1^{\lambda}}+4 n x^{2^{\lambda}}+\ldots . .+4 n x^{\left(\frac{n-2}{2}\right)^{\lambda}}+3 n x^{\left(\frac{n}{2}\right)^{\lambda}}$, when $\mathrm{n}$ is even and $\mathrm{n} \geq 4$.

and the generalized detour polynomial of four regular graph $\mathrm{G}\left(\mathrm{C}_{\mathrm{n}}\right)$ is,

$D_{\lambda} P(G: x)=\frac{n(n-1)}{2} x^{(n-1)^{\lambda}}$

\section{Corollary 3.1.2:}

Let $\mathrm{G}$ be the four regular graph. Then

The Wiener index $W_{1}(G)=\frac{n^{2}(n+1)}{2}$; The Reciprocal - Wiener index $W_{-1}(G)=-\left[\frac{n^{2}(n+1)}{2}\right]$;

The Harary - Wiener index $W_{-2}(G)=-\left[\frac{n^{2}(2 n+2)}{2}\right]$; The Hyper - Wiener index $W W(G)=\left[\frac{3 n^{2}(n+1)}{4}\right]$.

\section{Corollary 3.1.3:}

Let $\mathrm{G}$ be the four regular graph, then

The Detour index $D_{1}(G)=\frac{n(n-1)^{2}}{2} ;$ The Reciprocal index $D_{-1}(G)=-\left[\frac{n(n-1)^{2}}{2}\right]$,

The Harary- Detour index $D_{-2}(G)=-2\left[\frac{n(n-1)^{2}}{2}\right]$; 
The Hyper-Detour index $D D(G)=\left[\frac{3 n(n-1)^{2}}{4}\right]$.

\section{Theorem 3.1.4:}

Let $\bar{G}$ be the complement of four regular graph G. Then the generalized wiener polynomial and detour polynomial for $\bar{G}$ are respectively given by:

$$
W_{\lambda} P(\bar{G}: x)=\left(2 n^{2}-5 n\right) x^{1^{\lambda}}+4 n x^{2^{\lambda}} ; D_{\lambda} P(\bar{G}: x)=\left(2 n^{2}-n\right) x^{(2 n-1)^{\lambda}}
$$

\section{Proof:}

Let $\mathrm{G}$ be the four regular graph and $\bar{G}$ be the complement of G. Figure 1.16. shows the complement graph $\bar{G}$ of four regular graph $\mathrm{G}\left(\mathrm{C}_{4}\right)$. The wiener detour matrix Figure 1.17. gives the wiener polynomial and the detour polynomial of complement of four regular graph $\mathrm{G}\left(\mathrm{C}_{4}\right)$.

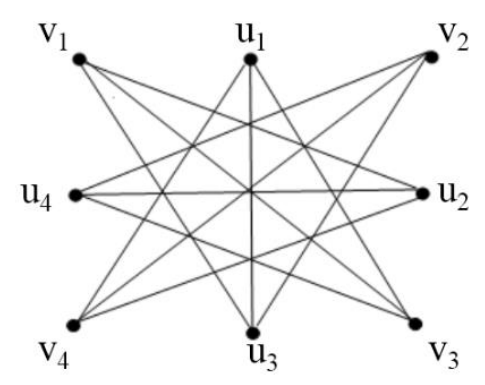

Figure 1.16. $\bar{G}$ of four regular graph $G\left(C_{4}\right)$

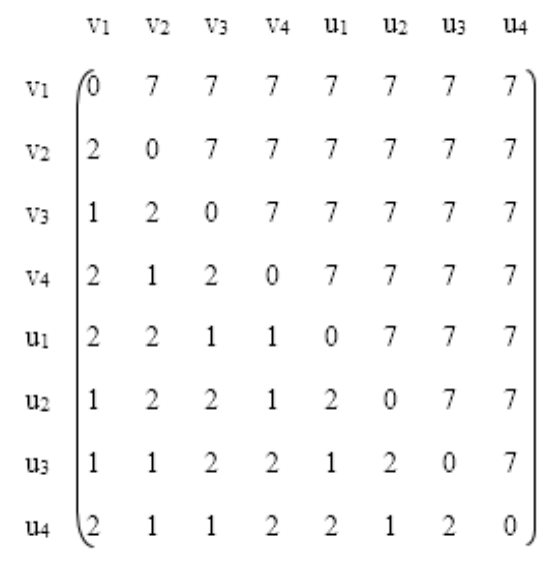

Figure 1.17.WDM[ $\left.\bar{G}\left(\mathrm{C}_{4}\right)\right]$

$W_{\lambda} P(\bar{G}: x)=12 x^{1^{\lambda}}+16 x^{2^{\lambda}} ; D_{\lambda} P(\bar{G}: x)=28 x^{7^{\lambda}}$

In similar mannar, when $\mathrm{k}=5,6,7,8$, the corresponding wiener polynomials of complement graph $\bar{G}$ of four regular graph given below,

$W_{\lambda} P(\stackrel{-}{G}: x)=25 x^{1^{\lambda}}+20 x^{2^{\lambda}} ; W_{\lambda} P(\stackrel{-}{G}: x)=42 x^{1^{\lambda}}+24 x^{2^{\lambda}} ; W_{\lambda} P(\stackrel{-}{G}: x)=63 x^{1^{\lambda}}+28 x^{2^{\lambda}} ;$

$W_{\lambda} P(\bar{G}: x)=88 x^{1^{\lambda}}+32 x^{2^{\lambda}}$

Hence in general, the generalized wiener polynomial of complement of four regular graph,

$W_{\lambda} P(\stackrel{-}{G}: x)=\left(2 n^{2}-5 n\right) x^{1^{\lambda}}+4 n x^{2^{\lambda}}$

In similar mannar, when $\mathrm{k}=5,6,7,8$, the corresponding generalized detour polynomials of complement graph $\bar{G}$ of four regular graph given below, 
$D_{\lambda} P(\stackrel{-}{G}: x)=45 x^{9^{\lambda}} ; D_{\lambda} P(\stackrel{-}{G}: x)=66 x^{11^{\lambda}} ; D_{\lambda} P(\stackrel{-}{G}: x)=91 x^{13^{\lambda}} ; D_{\lambda} P(\stackrel{-}{G}: x)=120 x^{15^{\lambda}}$

Hence in general, the generalized detour polynomial of complement of four regular graph is.

$D_{\lambda} P(\stackrel{-}{G}: x)=\left(2 n^{2}-n\right) x^{(2 n-1)^{\lambda}}$.

\section{Corollary 3.1.5:}

Let $\bar{G}$ be the complement of G. Then

The Wiener index $W_{1}(\bar{G})=\left[2 n^{2}+3 n\right]$; The Reciprocal index $W_{-1}(\vec{G})=-\left[2 n^{2}+3 n\right]$;

The Harary - Wiener index $W_{-2}(\stackrel{-}{G})=-2\left[2 n^{2}+3 n\right]$; The Hyper - Wiener index $W W(\stackrel{-}{G})=\frac{1}{2}\left[6 n^{2}+9 n\right]$

\section{Corollary 3.1.6:}

Let $\bar{G}$ be the complement of $\mathrm{G}$. Then

The Detour index $D_{1}(\vec{G})=4 n^{3}-4 n^{2}+n$; The Reciprocal - Wiener index $D_{-1}(\vec{G})=-\left[4 n^{3}-4 n^{2}+n\right]$;

The Harary- Detour index $D_{-2}(\bar{G})=-2\left[4 n^{3}-4 n^{2}+n\right]$; The Hyper - Detour index $D D(\bar{G})=\frac{12 n^{3}-12 n^{2}+3 n}{2}$.

Result 3.1.7: Nordhaus - Gaddum Equations of four regular graph.

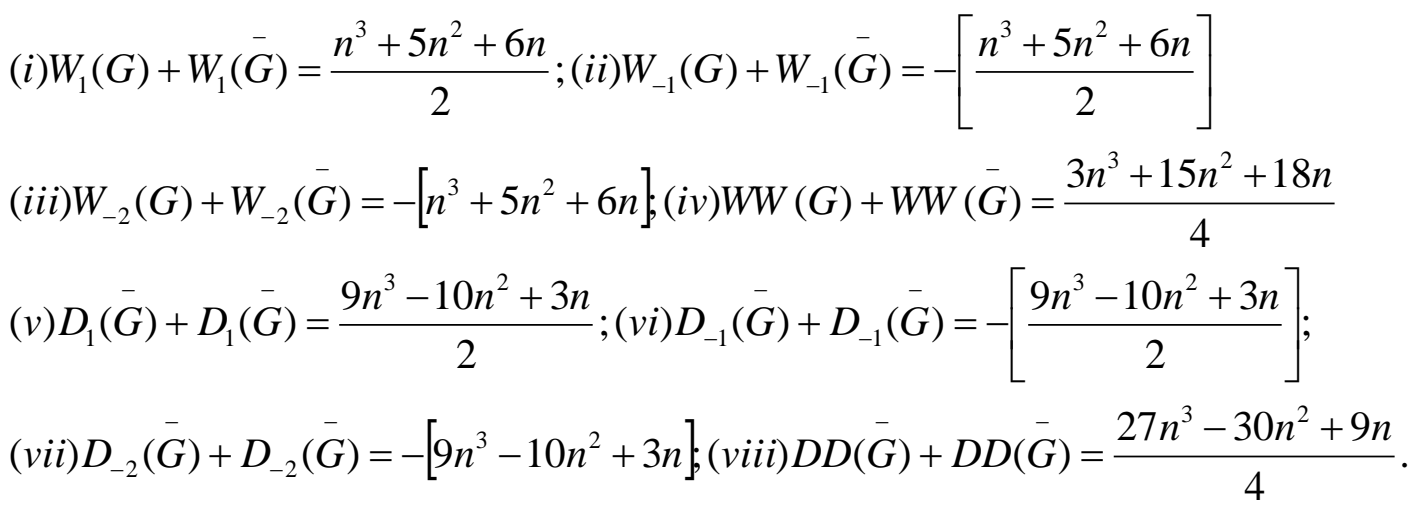

\section{Conclusions:}

In 1956, Nordhaus E. A., Gaddum J. W. [7] introduced the bounds involving the chromatic number $\chi(\mathrm{G})$ of a graph $\mathrm{G}$ and its complement. Many authors studied $[8,9]$ Nordhaus-Gaddum bounds for domination number, connected domination number, total domination number and also there has been many publications on Nordhaus-Gaddum type results for indices like Gutman wiener index, Steiner index, Krichhoff index. This paper deals with Nordhaus - Gaddum equations for wiener like indices to $\mathrm{k}$ - sun graph four regular graph. 


\section{References:}

[1] Diudea M. V., Indices of reciprocal properties on Harary indices, J. Chem.Inf. Comput. Sci. 37(1997), pp:292-299.

[2] Gutman I., A property of the Wiener number and its modifications, Indian J. Chem., 36A(1997), pp:128-132.

[3] Gutman I., Dobrynin A.A., Klavzar S., Pavlovic L., Wiener type invariants of tree and their relation, Bull Inst. Combin. Appl. 40(2004), pp:23-30.

[4] Joshi A., Baskar babujee J., Wiener Polynomial for Graphs with Cycle, Proceedings of the International Conference on Mathematics and Computer Science, (2008) pp:119-125

[5] Klavzar S., Zigert P., Gutman I., An algorithm for the calculation of the hyperWiener index of benzenoid hydrocarbons, Compt. Chem. 24(2000), pp:229-233

[6] Lukovits I., A note on a formula for the hyper - Wiener of some trees, J. Chem. Inf. Comput. Sci. 34(1994), pp:1079-1081.

[7] Nordhaus E. A., Gaddum J.W., On complementary graphs, Amer. Math. Monthly 63(1956), pp: 175-177.

[8] Payan C., Xuong N. H., Dominationbalanced graphs, J. Graph Theory 6 (1982), pp: 23-32.

[9] Plesnik J., Bounds on chromatic numbers of multiple factors of complete graph, J. Graph Theory 2 (1978), pp: 9-17. 